\title{
Carcinogenicity Screening of Chemicals Using Positron Annihilation Spectroscopy
}

\author{
Stepanov S.V.. ${ }^{1,2,3}$, Dubov L.Yu. ${ }^{1,2}$, Akmalova Yu.A. ${ }^{1}$, Byakov V.M. ${ }^{2,3,4}$, \\ Shtotsky Yu.V. ${ }_{0}^{1,2}$, and Bokov A.V.2,3 \\ ${ }^{1}$ National Research Nuclear University MEPhl (Moscow Engineering Physics Institute), \\ Kashirskoe shosse 31, Moscow, 115409, Russia \\ ${ }^{2} \mathrm{NRC}$ "Kurchatov Institute", Institute of Theoretical and Experimental Physics, Moscow Russia \\ ${ }^{3}$ Lomonosov Moscow State University, Moscow, Russia \\ ${ }^{4}$ D. Mendeleyev University of Chemical Technology of Russia, Moscow, Russia
}

\section{Abstract}

Chemical carcinogens are the main cause of cancer. Many thousands of new chemicals are synthesized annually in the world, unknown part of them is carcinogens. There are several methods, which are currently used for testing carcinogenicity. The fastest are

Corresponding Author:

S.V. Stepanov

stepanov@itep.ru

Received: 17 January 2018

Accepted: 25 March 2018

Published: 17 April 2018

Publishing services provided by Knowledge $\mathrm{E}$

(c) Stepanov S.V. et al. This article is distributed under the

terms of the Creative Commons

Attribution License, which

permits unrestricted use and

redistribution provided that the

original author and source are credited.

Selection and Peer-review under the responsibility of the PhysBioSymp17 Conference Committee.

\section{S OPEN ACCESS} physicochemical methods. We suggest the method of fast carcinogen screening using the positron annihilation spectroscopy (PAS). It is based on the fact that the presence of carcinogens, being strong electrophiles, significantly decreases positronium (Ps) formation probability. This quantity can be measured by means of conventional PAS techniques: positron annihilation lifetime spectroscopy (PALS), angular correlation of annihilation radiation (ACAR) or Doppler broadening of annihilation radiation (DBAR). It is also possible to evaluate anti-carcinogenic properties of chemicals using the anti-inhibition effect of Ps formation.

Keywords: carcinogen, electrophilicity, positron annihilation spectroscopy, positronium

\section{INTRODUCTION}

Chemical carcinogens are the main cause of cancer [1-3]. Many thousands of new chemical compounds are synthesized annually in the world, unknown part of which is carcinogens. New dangerous compounds may enter a human body with water, food, etc., then they dissolve in the aqueous or lipid media, integrate into the biochemical processes inside the cells, violate the mechanisms of self-regulation of cells and lead to their degeneration into malignant tissue. There are several methods, which are currently used for testing the carcinogenicity of chemicals (screening of carcinogens): 
epidemiological studies; experiments on animals; short-term tests (Ames test); correlation of molecular structure and its biological activity (QSAR - quantitative structure activity relationship); physicochemical methods.

The most reliable data is provided by experiments on animals. However, they are long-lasting and allow testing only a small part of the synthesized substances. The fastest are the physicochemical methods.

James and Elizabeth Miller established that almost all known carcinogens possess the common property of being highly electrophilic $[4,5]$. They described electrophile as a particle with vacant electron orbital (like $\mathrm{H}^{+}$or $\mathrm{Ag}^{+}$). These electron-deficient particles may interact with nucleophilic molecules (like $\mathrm{NH}_{3} \mathrm{Cl}^{-}$and deoxyribonucleic acid (DNA)) having unshared electron pairs.

Interaction between nucleophile $\left(: \mathrm{NH}_{3}\right)$ and electrophile $\left(\mathrm{H}^{+}\right)$results in association of reagents (formation of $\mathrm{NH}_{4}{ }^{+}$). The association is usually reversible. Only highly electrophilic chemicals form with nucleic acids undissociated complexes (like $\mathrm{Ag}: \mathrm{Cl}$ ), arising by combination of $\mathrm{Ag}^{+}$and $\mathrm{Cl}^{-}$). Such complexes cause mutations. High electrophility is a basic requirement to produce mutations. The Millers consider mutations as the first step of carcinogenesis, but for highest organisms not always sufficient to initiate neoplastic event $[4,5]$.

It is important to note that only some carcinogens originally have a positive electron affinity. The majority of chemical carcinogens (called procarcinogens) become active upon entering an organism after metabolism to ultimate forms through the oxidative action of enzymes.

The second starting point of Ps method is the idea which belongs to George Bakale. He supposed that the electrophility of chemical carcinogens may mean that they are able to scavenge efficiently excess quasi-free electrons that are produced in a nonpolar solvent by a short pulses of ionizing radiation. This property allows to identify carcinogens. In the presence of an electrophilic solute $S$, the high-mobility electrons will attach to the electrophile and thereby will be converted to low-mobility anions. This change in mobility of the charge-carriers is readily monitored and an electronattachment rate constant, $k\left(e^{-}+\mathrm{S}\right)$, can be extracted from the decay kinetics of the conductivity of the solution. A diffusion-controlled rate constant, which indicates the absence of a thermodynamic barrier in the electron-solute interaction, may be considered as a positive indication that the solute is an electrophile and a carcinogen, whereas a less-than diffusion controlled rate constant may be regarded as a negative indication of electrophility and carcinogenicity. 
Bakale's group for more than 10 years developed a pulse-conductivity method for fast detection of carcinogenic compounds. The essence of the method is as follows. A liquid cyclohexane was chosen as a model of the intracellular milieu. After the investigated substance was dissolved in cyclohexane, the solution was irradiated by nanosecond pulses of accelerated electrons. The slowing down electrons produced in the solvent a large number of ion-electron pairs. Before recombining with counter-ions, the thermalized secondary electrons had a chance to react with the solute $S$ and the reaction rate constant, $k\left(e^{-}+S\right)$, was measured through the electroconductivity induced in the solution.

Among all reactions of track electrons with chemicals identified as carcinogens in animal-test or epidemiological studies the reaction with tetrachloride $\mathrm{CCl}_{4}$, was characterized by the lowest diffusion controlled rate constant, $\mathrm{k}\left(\mathrm{e}^{-}+\mathrm{CCl}_{4}\right)$. Therefore potential carcinogenic hazard of a compound $S$ was quantified with the help of the inequality: $k\left(e^{-}+S\right)>k\left(e^{-}+C \mathrm{Cl}_{4}\right)$. If the rate constant $k\left(e^{-}+S\right)$ of the reaction between a solute $\mathrm{S}$ and a track electron was larger than the rate constant $k\left(\mathrm{e}^{-}+\mathrm{CCl}_{4}\right), \mathrm{S}$ was considered as a potential carcinogenic compound.

First measurements of Bakale's group have demonstrated impressive results [6]. From more than 40 compounds for which their rate constants with track electron exceed the rate constant of tetrachloride, only 5 do not belong to carcinogens according to biological criteria. From 18 compounds with rate constants lower than that of tetrachloride, 3, nevertheless, belong to carcinogens. Thus, the predictiveness of Bakale's method for carcinogenicity is more than $80 \%$. This is quite good accuracy index. An important distinctive feature of Bakale's method is its ability to detect not only ultimate but procarcinogens too.

The experimental results of Bakale's group have been recognized as rather promising. However, a practical realization of this method is not simple. The main part of Bakale's nanosecond pulse radiolysis setup constitutes an electron accelerator. It is clear that high cost of this facility, difficulties of maintenance, radiation shielding and disposal strongly narrow widely usage of pulse conductivity technique. Therefore, it seems reasonable to search possible improvements of Bakale's method. One of its modifications is discussed below.

We suggest an alternative method of fast carcinogens screening using the positron annihilation spectroscopy. This method is based on the following two facts: 1) formation of positronium (Ps) atom in a condensed medium proceeds as a result of combination of a thermalized positron with one of the quasi-free electrons of the 
positron track; 2) carcinogenic substances, being very strong electrophiles, are effective scavengers of quasi-free track electrons. Therefore, the presence of carcinogens significantly reduces Ps formation probability. To measure this probability and predict carcinogenic hazard of the tested substances, one may use standard techniques of positron annihilation spectroscopy [7]. The proposed method also allows to estimate anticarcinogenic properties of chemical compounds by the presence of an antiinhibition effect of Ps formation [8].

\section{MATERIAL AND EXPERIMENTAL PROCEDURES}

The study of the defect structure of matter using the positron annihilation is based on measuring the positron lifetimes in matter, changes in the energy of annihilation gamma quanta, or in the direction of their emission. The subjects of the study usually are the electron density of the material, the type and concentration of defects, the chemical environment of the positron annihilation point, the changing of these parameters under various external influences.

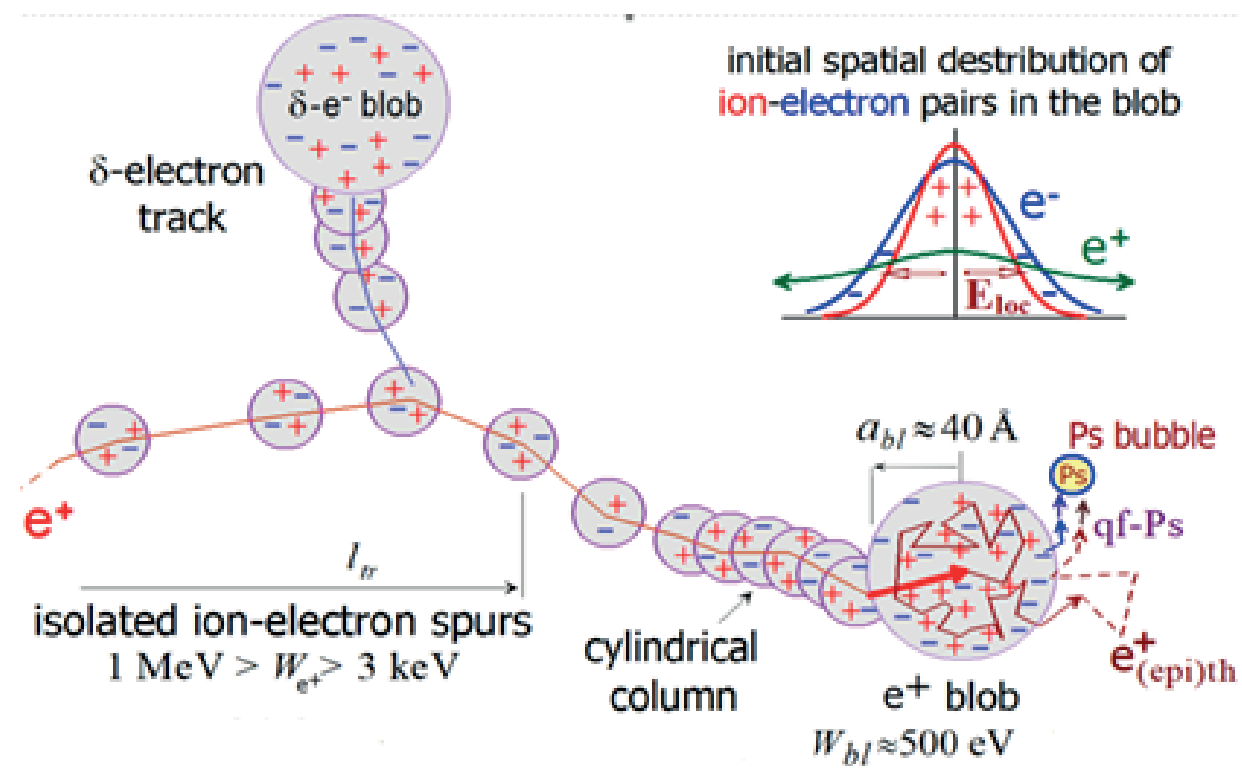

Figure 1: Scheme of the positron track. It consists of isolated ion-electron spurs, tracks of knocked out $\delta$-electrons, cylindrical column and the positron blob. Possible process of the Ps atom formation is also shown.

Entering a liquid, a fast positron looses energy $\left(\mathrm{W}_{e+}\right)$ in ionization collisions with molecules. At the end of its track, in its blob (which is a nanoscale spherical region of about 8-10 $\mathrm{nm}$ in diameter) it looses the rest of energy ( $\approx 500 \mathrm{eV})$ and creates 30 40 closely situated radical-cations $\left(\mathrm{H}_{2} \mathrm{O}^{+}\right)$- electron pairs, Fig. 1. Recombining with 
thermalized, but not yet solvated electrons, radical-cations may regenerate water molecules or form radiolytic hydrogen and $\mathrm{OH}$ radicals [9]:

$$
\mathrm{H}_{2} \mathrm{O}^{+}+\mathrm{e}^{-}=>\mathrm{H}_{2} \mathrm{O}^{*}=>\mathrm{H}_{2} \mathrm{O}, \mathrm{H}_{2}, \mathrm{OH}
$$

In the terminal blob the positron competes with radical-cations $\mathrm{H}_{2} \mathrm{O}^{+}$for the presolvated electrons. If it is succeeded to capture one of them, the positronium atom may be formed:

$$
\mathrm{e}^{+}+\mathrm{e}^{-}=>\text {ortho-Ps or para-Ps (reaction rate constant is } k_{e p} \text { ) }
$$

When scavenger $S$ of presolvated track electrons is added into solution in concentration $c_{S}$, the reaction:

$$
\mathrm{e}^{-}+\mathrm{S}=>\mathrm{S}^{-},\left(\text {reaction rate constant is } k_{e S}\right)
$$

competes for track electrons, see reactions (1-2), and suppresses yields of radiolytic hydrogen and Ps atoms [9]:

$$
\frac{P_{P S}^{0}}{P_{P S}\left(C_{S}\right)}=1+q_{S}{ }^{P_{s}} C_{S} \quad q_{S}^{P_{S}} \approx \frac{k_{e S} V_{0}}{k_{e p} n_{0}}
$$

Here $P_{P_{S}}{ }^{0}$ and $P_{P_{S}}\left(c_{S}\right)$ are the yields of $\mathrm{Ps}$ in the pure solvent and when $\mathrm{S}$ is added, $q_{S}{ }^{P S}$ is the inhibition coefficient of Ps formation; $V_{0}$ is the typical volume of the terminal $\mathrm{e}^{+}$blob and $n_{0}$ is the initial number of ion-electron pairs in it.

Ps formation can be easily observed.

When positrons annihilate with the electrons in the sample, in the most cases two gamma quanta are created. They are emitted in almost opposite directions. Angular deviation from collinearity is caused by the momentum of the electron. Hence, measuring the angular correlation of the annihilation radiation yields information about the momentum distribution of the electrons. Atomic electrons have a wide range of momentum. Therefore, the angular distribution of gamma quanta is rather wide. The momentum of an electron in a positronium atom is substantially less than that of atomic electrons. It leads to the appearance the narrow component in the angular correlation of annihilation radiation (ACAR) spectra (Fig.2a).

The formation of positronium can also be observed using the positron annihilation lifetime spectroscopy (PALS). While the lifetime of a positron in matter varies in the range of 100-500 ps, positronium in a triplet state (o-Ps) can live 10-100 times longer, that leads to the appearance of the long-lived component in the PAL spectra. Fig. $2 \mathrm{~b}$ schematically demonstrates transformation of the positron lifetime spectra when o-Ps formation probability varies from o to $25 \%$. 

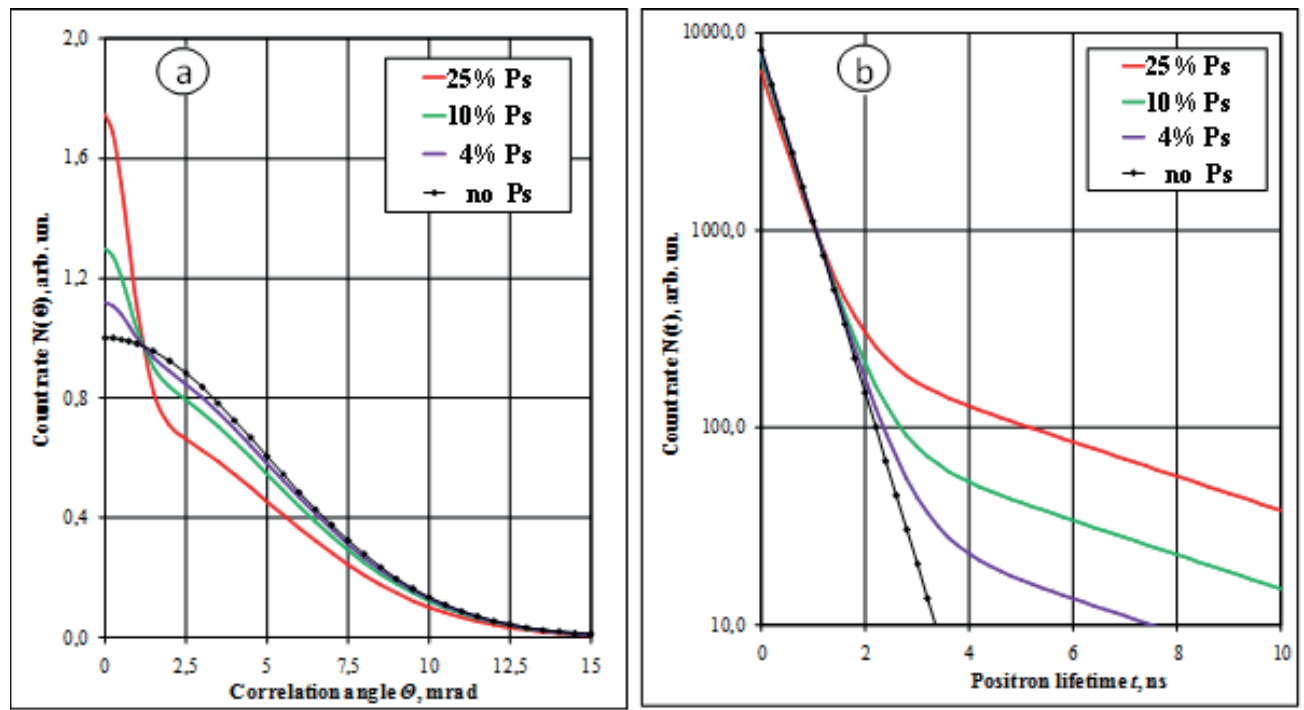

Figure 2: Typical shape of the ACAR (a) and PALS spectra (b) when Ps formation probability varies from o to $25 \%$.

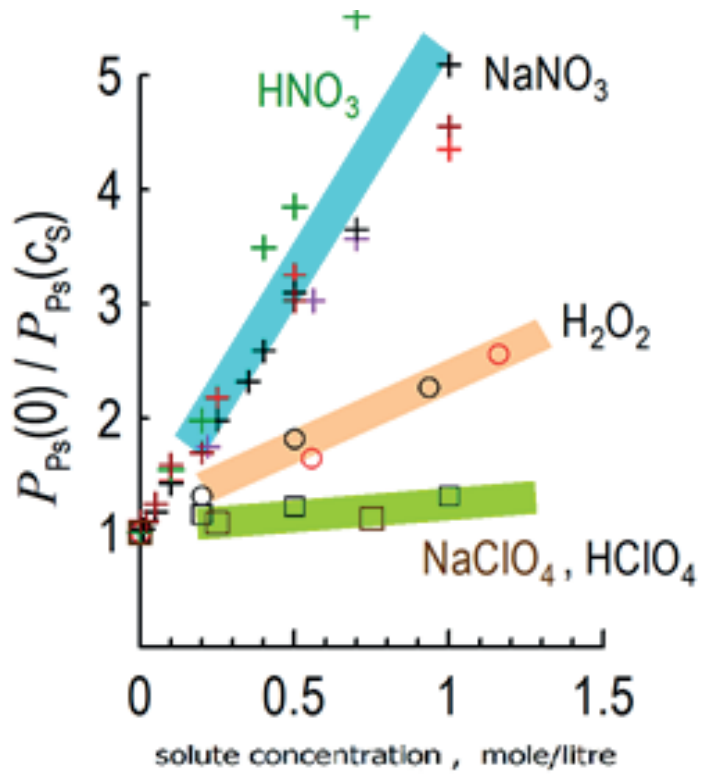

Figure 3: Dependencies of reciprocal yields of $\mathrm{H}_{2}$ and Ps in aqueous solutions of different electron scavengers vs. their concentrations [10].

\section{RESULTS AND DISCUSSION}

If the substance $S$ is an efficient electron scavenger, it can suppress Ps formation, if its concentration is large enough. As we have mentioned above, efficient e- scavengers are very probably carcinogens. Therefore, a negligible Ps yield in a solution of the testing substance $S$, indicates that $S$ could be a carcinogen.

Fig. 3 illustrates Eqs. 4 for different electron scavengers in aqueous solutions. It is clear from this approach that if a substance $S$ is an efficient electron scavenger, it 
almost completely suppress formation of the Ps atom, if its concentration is enough large. But as we mentioned above, efficient electron scavengers are very probably carcinogens. Therefore, a negligible yield of $\mathrm{Ps}$ in a solution containing a compound $\mathrm{S}$, indicate that this substance could be a carcinogen. This is the idea of the positron annihilation method of detecting potential carcinogens.

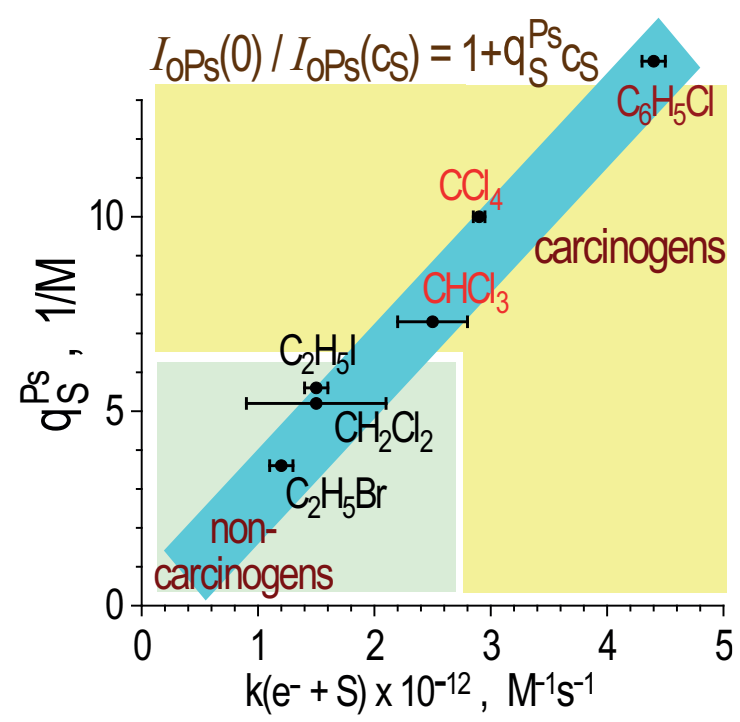

Figure 4: Inhibition coefficients of Ps formation in cyclohexane by different electron scavengers vs. reaction rate constants $\mathrm{k}(\mathrm{S}+\mathrm{e}-)$.

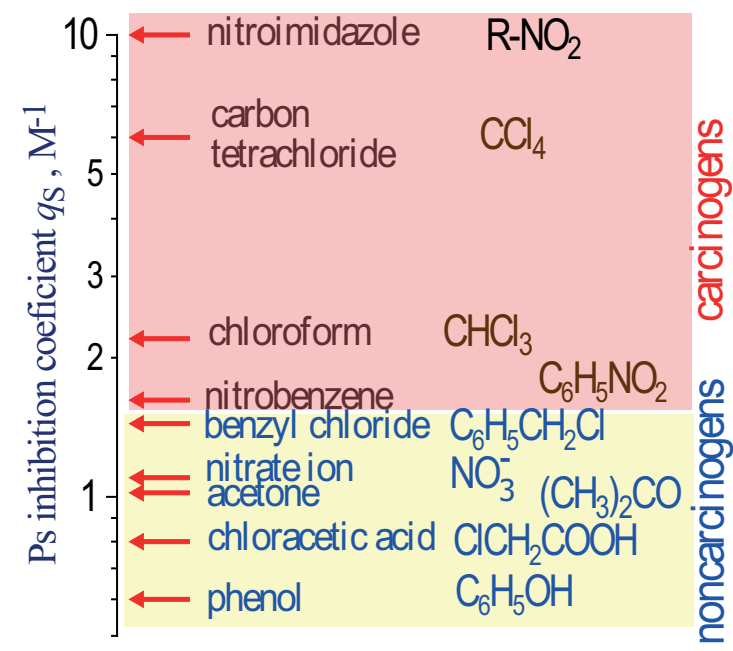

Figure 5: Inhibition coefficients of the Ps yield by different track e-scavengers dissolved in ethanol.

Tests of several dozens (carcinogens and non-carcinogens) confirmed the efficiency of the positron method [11]. Fig. 4 demonstrates a rather good correlation between Ps formation inhibition coefficients $q_{S}$ in cyclohexane by different scavengers $S$ of quasifree track electrons. The strongest Ps inhibitors $\left(q_{S}>6 \mathrm{M}^{-1}\right)$ are the most efficient e- scavengers, $k_{e S}>3 \cdot 10^{12} \mathrm{M}^{-1} \mathrm{~s}^{-1}$ and according to biological data they are carcinogens. Electrophylic molecules with large Ps inhibition coefficients, $q_{S} \geq 2 \mathrm{M}^{-1}$, 
are also carcinogens. Polar media could be considered as an appropriate model of intracellular milieu for DNA molecules, so measurements have been carried out in ethanol solutions (Fig. 5). Fig. 6 shows the probability of ortho-Ps formation and the reaction rate constant with hydrated electrons for various chemical compounds in their pure form. It can be seen that the probability of Ps formation does not exceed $3 \%$ in chemical substances possessing carcinogenic properties, confirmed in tests with animals.

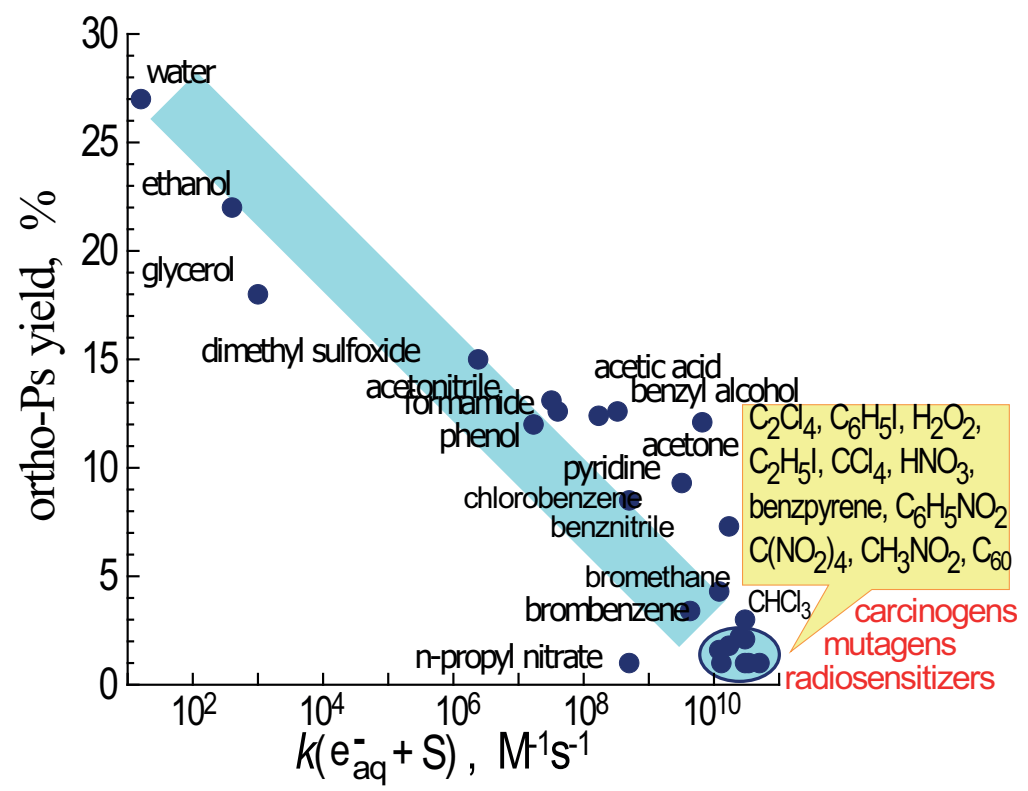

Figure 6: Ortho-Ps yields their reaction rate constant with hydrated electrons in various chemical compounds.

As we said above, the yield of positronium can be measured by different methods of positron annihilation spectroscopy: positron annihilation lifetime spectroscopy (PALS), Doppler broadening of annihilation radiation (DBAR) and angular correlation of annihilation radiation (ACAR). To choose the most appropriate for evaluating carcinogenicity method, the performance characteristics of PAS techniques must be taken into account, the most important of which are given in Table. 1.

From the above characteristics it can be seen that the most suitable platform for the development of a laboratory test procedure is the PALS-spectrometer. This method has high accuracy, uses a low active source of positrons, can work for a long time in an autonomous mode; it is possible to automate the processes of collecting and processing information. A significant exposure time, which is its main drawback, can be overcome by increasing the number of registration channels. Even in the standard configuration, the life-time spectrometer, having small dimensions and mass, is placed on a laboratory table. After testing the method for evaluating the carcinogenicity of 
TABLE 1: Some characteristics of PAL spectrometers.

\begin{tabular}{|c|c|c|c|}
\hline & PALS & DBAR & ACAR \\
\hline Positron source activity & $\sim 10 \mathrm{MBq}$ & $\sim 10 \mathrm{MBq}$ & $\sim 1 \mathrm{GBq}$ \\
\hline Exposition time & $\sim 10$ hours & $\sim 2$ hours & $\sim 20$ hours \\
\hline $\begin{array}{c}\text { Accuracy of } \\
\text { positronium inhibition } \\
\text { estimation }\end{array}$ & high & medium & high \\
\hline Dimensions of device & Portable(desktop) & Portable(desktop) & Length 3-5 m \\
\hline Additional equipment & - & $\begin{array}{l}\text { Cooling system with } \\
\text { liquid nitrogen }\end{array}$ & $\begin{array}{l}\text { Reactor (the case of } \\
\text { using Cu-64 as a } \\
\text { positron source) }\end{array}$ \\
\hline $\begin{array}{l}\text { Ability to optimize } \\
\text { measurements }\end{array}$ & + & - & + \\
\hline Operating personnel & 1 person & 1 person & 2 persons \\
\hline
\end{tabular}

the substances on a standard spectrometer, it is possible to develope a specialized portable device.

\section{CONCLUSIONS}

PALS is able to detect electrophilicity of chemical carcinogens by their inhibition of positronium (Ps) formation in liquid media. The use of PALS technique in combination with other instrumental tests makes it possible to increase the efficiency of detection of carcinogens among chemicals in their mass screening. The proposed method also allows to evaluate anticarcinogenic properties of chemical compounds by the presence of an anti-inhibition effect of Ps formation.

In connection with this, we note another such interesting result. It turns out that in the cancer-affected skin of mice, the yield of Ps is abnormally low. This suggests that in the cells of such tissues there is an accumulation of a significant concentration of carcinogens. Thus, the positron method not only facilitates, accelerates and reduces costs of identifying carcinogens, but also opens up new opportunities in studies of carcinogenic action.

\section{ACKNOWLEDGMENTS}

This work was supported by the MEPhl Academic Excellence Project (Contract No. 02.003.21.0005). 


\section{References}

[1] J. Ferlay et al. Estimates of worldwide burden of cancer in 2008: GLOBOCAN 2008. Int J Cancer 127(12): p. 2893-917, 2012.

[2] ACS, Cancer facts \& figures 2015. 2015, American Cancer Society: Atlanta.

[3] D. Jollow, Biological reactive intermediates: formation, toxicity, and inactivation, Springer 2013.

[4] Miller J. A., Miller E.C. J. Nat. Cancer Inst. 47 v-xiv (1971)

[5] Lovelock I.E., Zlatkis A., Becker R.S. Affinity to polycyclic aromatic hydrocarbons for electrons with thermal energies: its possible significance in carcinogenesis. Nature. V. 193 , No. 4815 , P. 540,1962

[6] Bakale G., McCreary R.D., Gregg E.C. "Quasifree electron attachment to carcinogens in liquid cyclohexane". Cancer Biochem. Biophys., v.5, 103 (1981)

[7] Byakov V.M., Stepanov S.V., Stepanova O.P., Positron Method for Diagnostics of Carcinogens, Material Science Forum, v. 607, p. 223 (2009)

[8] Byakov V.M., Stepanov S.V., Stepanova O.P. "PAL spectroscopy and testing for potential carcinogens", Physica Status Solidi (C) Current Topics in Solid State Physics, v. 6(11), p. 2503, 2009.

[9] Byakov V.M. J. Int. Radiat. Phys. Chem., v. 8 (3), p. 283 (1976); J. de Phys., IV, Colloque C4, Supplement II, v. 3 (1993) and references therein

[10] Byakov V.M., Grafutin V.I. Radiat. Phys. Chem., v. 28 (1), p. 1-18 (1986)

[11] Byakov V.M., Stepanov S.V., Stepanova O.P., Biannual Journal of Japanese Society of Radiat. Chem. v.100, p.46 (2015) 